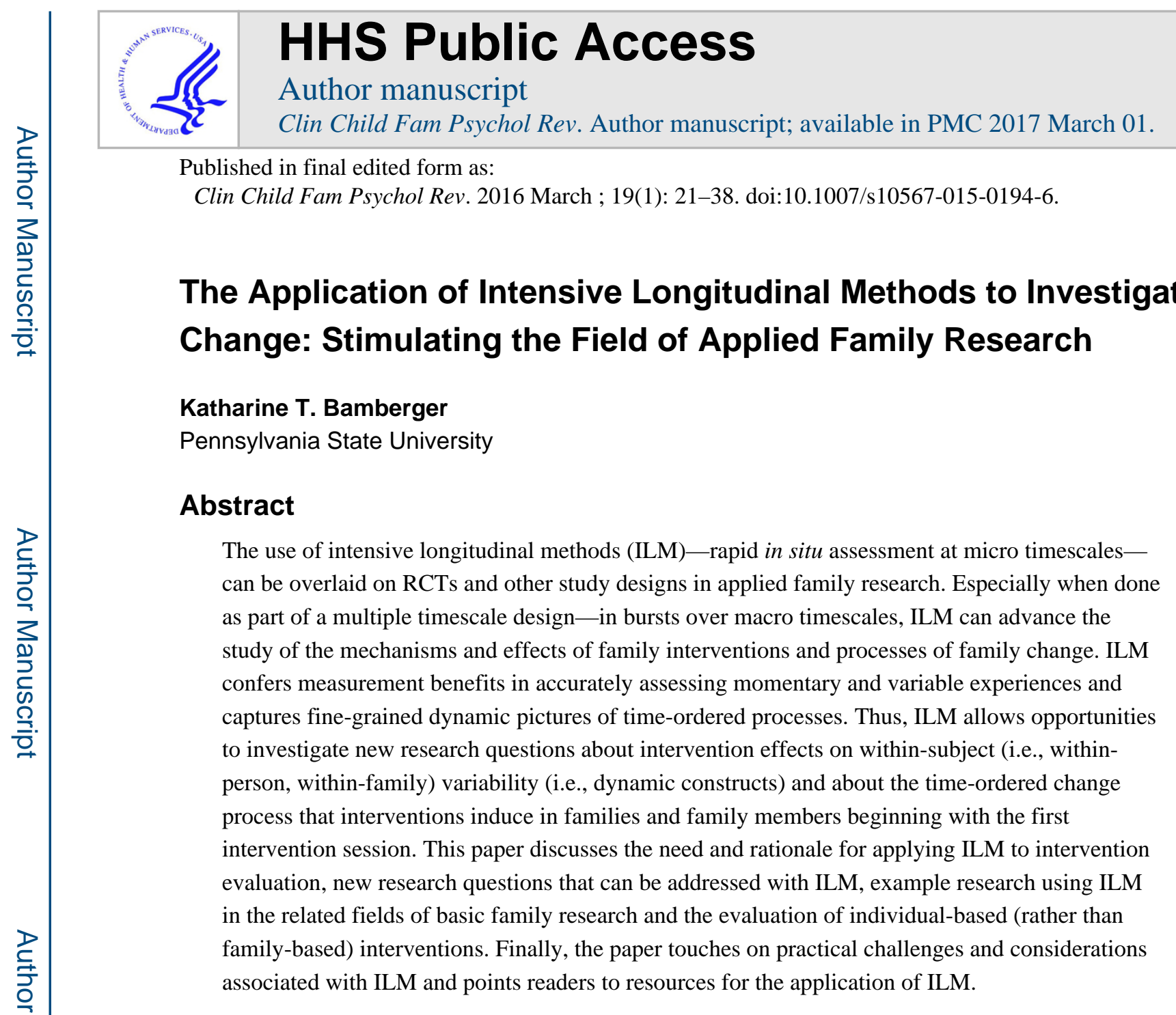

\title{
Keywords
}

intensive longitudinal methods; ecological momentary assessment; applied family research; family intervention; program evaluation; prevention science; multiple timescale design

Consider the gold standard study design for evaluating the efficacy of an intervention with families - a randomized controlled trial (RCT) with standardized baseline and postintervention assessments. In it, first, family members are assessed with questionnaires and sometimes observed to ascertain their baseline levels of functioning. Questionnaires are usually retrospective, asking family members to determine a single rating summarizing experiences in the last few months on indices of interactions, feelings, and/or behavior. These questionnaires give researchers a sense of family members' functioning in broad strokes-general experiences and characteristics of the person and family. Then, families are

\footnotetext{
Address correspondence regarding this manuscript to: Katharine Bamberger, Department of Human Development \& Family Studies, Pennsylvania State University, 119 Health and Human Development Building, University Park, PA 16802, USA or ktb5128@ psu.edu. Author notes: Katharine T. Bamberger, M.S., Doctoral Candidate, Dept. of Human Development and Family Studies; Bennett Pierce Prevention Research Center

Disclosure Statement

The author has no conflicts of interest to declare.
} 
randomized to conditions, including the treatment of interest and a control condition. After the intervention has concluded, family members are again assessed with questionnaires and observations to ascertain their current (post-intervention) level of functioning. This allows for evaluation macro timescale change-over months and years-in their general experiences and characteristics. Family members may be assessed numerous times after the intervention, usually after six months or more, or at developmental transitions to ascertain later levels of functioning. This assessment schedule has served the field well for addressing the overall efficacy of interventions programs based on group comparisons and change on a macro developmental timescale. For example, questions like-does the intervention group have a better developmental outcome than the comparison group on characteristic levels of affective quality—can be addressed with this assessment schedule. But, what additionally important information can be gained from extending the RCT design with other assessment methods? The current paper will demonstrate the substantial value that incorporating intensive longitudinal methods (ILM) - in situ assessment at a micro timescale including moments, interpersonal interactions and days-into applied family research studies can add to yield insights into how interventions work for families.

To do this, I first define ILM, reviewing protocols, and their use, and advantages. I then summarize our current understanding of the mechanisms of family intervention programs, highlighting limitations of the current methodologies. Third, I review theoretical perspectives that emphasize family processes of intervention change to illustrate the value of assessing families at micro timescales. Fourth, I propose research questions that could be addressed using ILM and that would increase the depth and specificity of evaluation of family interventions. Finally, I review research demonstrating the feasibility and added value of ILM in both basic family research and intervention evaluation. The goal of this paper is to spur intervention scientists across the treatment spectrum to consider utilizing ILM in study designs to better understand the process of change and how interventions work.

In this paper, I discuss the evaluation of family intervention programs. These are programs that engage parents and possibly additional family members to optimize family functioning and/or child development. I will be taking a broad perspective, encompassing all levels of intervention from universal prevention that invites all families to participate to treatment that aims to alleviate existing problems.

To answer the question posed earlier: what information can we gain from extending the traditional assessment schedule of the RCT with ILM, consider a second study with a different assessment schedule - a multiple timescale design overlaid on a RCT. Here, each family member reports on domains of daily life such as his/her own feelings, behavior, and interactions with other family members. Family members make these ratings based on the current day or current interpersonal interaction and do so in "bursts" of several days at baseline, during the intervention, and again post-intervention. Because these assessments occur frequently and close to the time of the actual experience, such data can be used to evaluate changes at a micro timescale and functional form of change that is not possible with traditional survey methods. 
As prevention and intervention sciences increase their focus on understanding how interventions work, ILM can be used to further investigate within-subject change processes. Advantages of using ILM to understand intervention effects are discussed further below.

\section{Intensive Longitudinal Methods}

This paper will use the terms intensive longitudinal methods (ILM), intensive repeated measure studies, and intensive longitudinal data as labels for rapid in situ assessment protocols, studies that employ these protocols, and the resulting data, respectively (Bolger \& Laurenceau, 2013). Intensive repeated measure studies use ILM to assess participants repeatedly in short intervals - often multiple times per day — conducting assessments in situ, as participants go about their lives. This method of assessment is ideal for capturing momentary experiences or characteristics, which Conner and Barrett (2012) refer to as the experiencing self. ILM encompass protocols including daily diaries, experience sampling, and ecological momentary assessment and can be used as a general term to describe any of these protocols (Stone \& Shiffman, 1994; Smyth \& Stone, 2003; Bolger, Davis, \& Rafaeli, 2003; Laurenceau \& Bolger, 2005).

The following is a review of various ILM protocols including their similarities, differences, and uses; see Table 1. Ecological momentary assessment refers to protocols that capture proximal experiences at a short micro timescale and can be contingent on intervals, events, or device signals-whichever theoretically matches the time course of the process being investigated (Stone \& Shiffman, 1994). For example, sampling can occur at specified intervals such as every two hours, or after a specified event such as the enactment of a family routine (Shiffman, 2007; Stone \& Shiffman, 1994). Experience sampling protocols, used since the 1970s, are device-contingent protocols notifying participants at random moments of the day to record their current experience (Csikszentmihalyi, Larson \& Prescott, 1977; Hektner, Schmidt, \& Csikszentmihalyi, 2007). Beyond self-report measures, technological advances have made additional modes of intensive data collection possible, including passive data collection, which is often referred to as ambulatory assessment (Society for Ambulatory Assessment, 2014). Ambulatory assessment can be conducted on event, interval, and device-contingent protocols and can collect many types of data such as global positioning system (gps) location, nearby Bluetooth devices and wi-fi networks, accelerometer data/actigraphy, ambient sound (i.e., electronically activated recorder; EAR), video or photo recording, cardiac activity, respiration, and skin conductance (Trull \& EbnerPriemer, 2009; 2013).

Daily diaries are completed once per interval, generally at the end of each day to assess thoughts, feelings, behaviors, and/or experiences from that day, making their micro timescale longer than many other protocols. They have been used to study marital and family relationships and stress among other topics (e.g., Birditt, Nevitt, \& Almeida, 2014; Grzywacz, Almeida, \& McDonald, 2002; Scott, Sliwinski, Mogle, \& Almeida, 2014; Sin, Graham-Engeland, \& Almeida, 2015).

ILM have become increasingly popular due to both new technologies to administer these assessments (Shiffman, 2007; Smyth \& Stone, 2003) and new statistical methodologies to 
fully exploit the intensive longitudinal data (Mehl \& Conner, 2012; Walls \& Schafer 2006). The major benefits of ILM are the measurement advantages that they confer, which make these methods ideal for investigating questions involving within-subject change and dynamic processes manifested as within-subject variability (Collins, 2006; Ram \& Gerstorf, 2009; Sliwinski, 2008). ILM have three main advantages compared with retrospective selfreport: (1) avoidance or minimization of retrospective recall bias (Stone, Shiffman, Atienza, $\&$ Nebeling, 2007), which is especially important for behaviors that are frequent and/or irregular and for assessing intensity of experience (Schwarz, 2007), (2) ecological validity of the assessment context, which is a benefit over laboratory task-oriented observational methods (Stone et al., 2007), and (3) specificity of temporal events, which allows the study of dynamic within-subject processes (Collins, 2006; Stone et al., 2007; Stone \& Shiffman, 1994). Because of these advantages, ILM provide temporally rich and specific data. See Table 2 for a summary of differences between ILM and retrospective assessment and the measurement and timescale benefits of ILM.

In family research, ILM allow an in-depth perspective on families' lives (Larson \& Almeida, 1999). These methods capture family interactions that happen within a given day—aspects of life that both directly contribute to development and are targeted in family interventions (Smyth \& Heron, 2014), yet which otherwise elude measurement. Because these interactions recur with variation many times within the usual time frames specified for questionnaires, it is often more difficult for participants to recall distinct interactions, and therefore information about each interaction is lost. Capturing these interactions by assessing individuals on a micro timescale is the added value of ILM - these methods allow estimation of rapid change or fluctuation in within-family and within-person (i.e., within-subject) processes.

In intervention evaluation using multiple-timescale designs, we can examine intervention effects on these dynamic characteristics and processes, which can only be captured by assessing individuals on a micro timescale. Here, I take a moment to define dynamic characteristics and processes (c.f., Ram \& Gerstorf, 2009). These outcomes are integral to the advancements that can be made by incorporating ILM in program evaluation designs. Dynamic characteristics can be thought of as short-term (e.g., moment-to-moment or day-today), fluctuations that may be characteristic of the person, his/her context or experience. They appear as time-unstructured (i.e., random) fluctuations. Conceptually, dynamic characteristics can be thought of as sensitivity, lability, robustness, or plasticity. For example, fluctuations in affect may represent lability while fluctuations in use of parenting skills/strategies may represent plasticity or willingness to try new strategies. Also important to evaluating family interventions, dynamic processes are the time-ordered processes of change viewed at a fine-grained timescale. Dynamic processes are smooth changes that can be modeled by equations (e.g., sine curves, quadratic functions). Conceptually, they yield insight into short-term change processes such as skills uptake, adaptation, and homeostasis.

ILM can increase our knowledge of intervention effects in three ways presented in Table 3. First, they allow researchers to test for intervention-related change in momentary experiences and dynamic characteristics and processes, i.e., new outcomes that encompass variability in those behaviors, feelings, and interactions that occur in daily family life 
(Robbins \& Kubiak, 2014). Second, ILM can chart intervention-related change as it occurs on the micro timescale and provide sufficient data to uncover the functional shape and causes of change and thus contribute to evidence of mechanisms (Bolger \& Laurenceau, 2013; Shiffman, Stone, \& Hufford, 2008). Comparisons among programs in the functional shape of change may also be useful. For example, a treatment where families begin changing as soon as the program begins and change more rapidly may be more desirable than a treatment where families reach the same level of change at the end of the study but have a delayed and slower rate of change. Third, ILM allow researchers to investigate which factors influence micro timescale change, including behavior, stable trait characteristics, and dynamic characteristics and processes. Although the benefits that ILM offer are substantial, these benefits are tempered with the realization that the use of ILM will increase participant burden by having more frequent, albeit brief, assessments. This may prove to be more costly, especially for higher-risk samples whose compliance in intensive assessment protocols may need to be closely monitored. The issue of burden is further discussed in the section on Challenges and Considerations.

\section{Evidence for Family Programs}

\section{Efficacy of Family Programs}

During the last 20 years there have been major advances in the evaluation and widespread dissemination of family prevention and treatment programs (IOM, 2009; Barrera \& Sandler, 2006; Mrazek \& Haggerty, 1994; Weisz, Sandler, Durlak, \& Anton, 2005; Spoth et al.,

2013). Reviews and meta-analyses show that programs work to prevent and treat a variety of outcomes (e.g., drug abuse, delinquent behavior) and promote competencies in a variety of populations (Barrera \& Sandler, 2006; Weisz et al., 2005; Durlak \& Wells, 1997; Curtis, Ronan, \& Borduin, 2004; Thomas \& Zimmer-Gembeck, 2007). However, the types of effects that have been evaluated have been limited to global outcomes (e.g., parents' monitoring behavior, discipline, affective quality of relationships), macro-time effects (e.g., changes from baseline to post-intervention, over months), and between-person and betweengroup comparisons (e.g., comparing those assigned to the intervention to those assigned to the comparison group). We know very little about the dynamic processes involved in change, the functional shape of micro timescale change due to interventions, within-subject influences (e.g., what impacts changes within persons across time) or about change in experiences and dynamic characteristics.

\section{Mechanisms of Family Programs}

Although the effectiveness of family programs is now well-documented, albeit in broad strokes, much less is known about how these evidence-based programs work. One way this has been pursued is through mediation analysis to uncover the mechanisms by which programs have their effects (Barrera \& Sandler, 2006; Kazdin, 2004; Sandler, Schoenfelder, Wolchik, \& MacKinnon, 2011; Weisz et al., 2005). Intervention mechanisms are those changes in cognitions, affect, and behavior that account for the intervention's effect on a certain outcome. For example, parent factors such as the quality and frequency of positive and hostile parent-child interactions, sensitivity, and behavioral control are mechanisms that 
can be targeted by intervening with parents to impact child development (Lamborn, Mounts, Steinberg, \& Dornbusch, 1991; Poulin, Nadeau, \& Scaramella, 2012).

The following is a brief account of work that contributes to our understanding of intervention mechanisms. Rather than a comprehensive review, this section highlights research that investigates intervention mechanisms. The Nurse-Family Partnership (NFP), a home-visiting program for first-time high-risk mothers targets key risk and protective factors in the family's life course. Three targeted risk factors; prenatal health behaviors, sensitive competent parenting, and parental life course decisions have been impacted in RCTs testing NFP's effects (Olds, 2012). While these risk factors are hypothesized to be mechanisms of NFP's effects on more distal factors, including later parental outcomes, child functioning, and child abuse and neglect injuries, their link with these distal factors has not been tested.

Variables that show statistical mediation are likely to be intervention mechanisms (unless they are proxies for processes that are not yet well understood). Recent mediation studies have revealed support or partial support for theories of the intervention and developmental theories of risk factors. Parent Management Training- Oregon Model (PMTO; Forgatch, 1994) is a parent intervention targeting parenting practices to ultimately impact child delinquency. PMTO is tested with divorced and single parents; divorce both confers risk associated with having a single parent and is a period of transition, making this sample ideal for efficacy trials. Mediation studies have shown that the intervention impacts parenting, and change in a global summary of parenting practices from baseline to year 1 was related to reductions in delinquency eight years later. Another model showed similar results for changes in parenting mediating year 9 arrest frequency (Forgatch, Patterson, Degarmo, \& Beldavs, 2009). Similarly, with Somali and Pakistani mothers living in Norway, baseline to post-intervention changes in positive parenting and reduction in harsh discipline mediated PMTO's effects on child conduct problems (Bjørknes, Kjøbli, Manger, \& Jakobsen, 2012). A limitation of this study was that parenting practices and child conduct problems were both assessed at the post-intervention time point. In another study, a theoretical model of cascading effects was tested, where PMTO was hypothesized to impact parenting, which would impact child behavior, which would impact parental depression, and these effects would cascade, affecting each other thereafter. Findings indicated that, changes in parenting from baseline to one year partially mediated latent growth in both internalizing and externalizing child behavior (DeGarmo, Patterson, \& Forgatch, 2004).

Family Check Up (FCU; Dishion \& Stormshak, 2007) is a tiered intervention targeting parenting practices and family functioning. Recent studies investigating multiple mediators and intervening variables of the FCU have found support for the following hypotheses using structural equation modeling. In one study, FCU improved family relationship quality over four years after the intervention which accounted for differences in parent monitoring and early sexual experience-- both of which were partial mediators of high-risk sexual behaviors across gender and race groups (Caruthers, Van Ryzin, \& Dishion, 2014). In a study of the mediators of FCU's impact on adolescent antisocial behaviors, change in family conflict over three years mediated the relation between change scores in parents' behavior concerns at one year and adolescent antisocial behavior three years later (Fosco, Van Ryzin, Stormshak, \& Dishion, 2014). In a report on FCU's impact on obesity in young adulthood, 
improvements in parent-child relationship quality predicted intervening differences in eating attitudes in late adolescence, which predicted obesity in young adulthood (Van Ryzin \& Nowicka, 2013). Healthy behaviors and depressive symptoms were found to not be mediating effects of obesity. These findings show the importance of mediation analysis for testing the theory of the intervention. However, these studies also show that studies are apt to miss measurements of intermediate change constructs (e.g., actual parenting behaviors at meal time) and possible indicators of time-varying individual differences (e.g., daily childhood eating attitudes and behaviors at meal time) due to absence of intensive data collection during the trial.

The above reviewed between-subject mediation studies illustrate recent findings on intervention mechanisms. These findings demonstrate that little is known about the process of change even when between-person mediators have been identified. Results of betweenperson mediation, while productive, may not correspond to mechanisms of change for an individual (Molenaar, 2004). Sandler and colleagues (2011) reviewed parenting programs that have assessed longer-term outcomes ( 1 year+) in order to evaluate whether changes in parenting that had been identified as intervention mechanisms were in fact mediating child outcomes. Results indicated that a wide range of parenting programs affected both long-term child outcomes and parenting. The authors concluded that still little is known regarding the theory and process of long-lasting change (i.e., series or cascade of mechanisms).

ILM may shed light on this process of long-lasting change; for example, changes in families' micro timescale interactions may accumulate and contribute to long-lasting change in family members and their relationships. ILM can be used to investigate within-subject processes that may mediate intervention effects (Heron \& Smyth, 2010).

Also important in influencing the change process is participants' active engagement in intervention programs. Learning can be influenced by personal characteristics and by factors outside of the intervention sessions like family members' reactions to new tactics learned in an intervention, both of which are not observable by practitioners. It is possible to capture the dynamic and unobservable aspects of engagement with ILM (e.g., practice, selfreflection, motivation, and evaluation of the intervention). This can allow investigators to understand what participants do, think, and feel over the duration of the intervention to influence their own processes of change (Bamberger \& Coatsworth, 2013; Bamberger, Coatsworth, Fosco, \& Ram, 2014).

\section{Theoretical Underpinnings}

In this section, I review the theoretical underpinnings and rationale for using ILM in applied family research. First, I connect the importance of theory-based momentary and dynamic micro timescale constructs to their assessment with ILM. Second, I discuss two conceptualizations of short-term within-subject change that can be evaluated using ILM: the functional shape of short-term change, and the cascade of mediating processes in withinsubject change. 


\section{Family Theory and Constructs on a Micro Timescale}

Dynamic Constructs in Developmental Theory-Families' everyday interactions are among the most direct (i.e., proximal and constant) contributions to development (Bronfenbrenner \& Morris, 1997; Sameroff \& MacKenzie, 2003) and are influenced by the development of each family member (Schermerhorn \& Cummings, 2008). Family interactions occur in micro timescales and accumulate over time, becoming of great importance to child outcomes (Bai \& Repetti, 2015). Thus, directly assessing these interactions in real time as they are experienced by parents and child may yield new insights into development (Bai \& Repetti, 2015; Laurenceau \& Bolger, 2005; Smyth \& Heron, 2014).

This idea of the importance of momentary experience is shared across multiple theoretical perspectives. Bronfenbrenner places the family at the central hub of the bioecological model, both influencing development directly from its own characteristics and processes and as the mediating factor for indirect effects of contextual influence (1986). In this formulation, Bronfenbrenner viewed the family as exerting influence through "family processes", such as parenting behaviors like reciprocal verbal interactions (1986, p.724). Bidirectional influences within a family system are often emphasized in system theories (Cox \& Paley, 1997; Minuchin \& Fishman, 1981; Sameroff \& Fiese, 2000). Family systems theory suggests that systems develop over micro timescales through amplifying and dampening variations in individuals' behavior and interactions; positive feedback amplifies and provides the possibility of novelty, and negative feedback dampens variations and tends to encourage stability (Granic, Dishion, \& Hollenstein, 2003). Modeling system processes is a promising avenue for uncovering developmental and intervention change processes in the family because new patterns of interaction may emerge, and this emergence can be modeled at the level of individual interactions over time (Fosco, Bumbarger, Bamberger, \& Van Ryzin, 2015; Granic, Dishion, \& Hollenstein, 2003; Schermerhorn \& Cummings, 2008). This can be done with ILM (Larson \& Almeida, 1999; Repetti, Reynolds, \& Sears, 2015) to provide a rich dynamic picture of the family change process (Shiffman, Stone, \& Hufford, 2008). In the ecocultural framework, everyday activities and routines both carry cultural meaning and are the natural units of experience for family members (Weisner, 2002). This framework suggests that family routines that are meaningful and consistent with the family's cultural values, fitting the family's resources, providing predictability, and balancing family members' interests/reducing conflict, will produce well-being (Weisner, 2010; Weisner, Matheson, Coots, \& Bernheimer, 2005).

Dynamic Constructs in Family Intervention Theory-In terms of the types of change interventions induce in families, interventions differ in their implicit (or sometimes explicit) theories of change. Two main possibilities include disrupting the family's homeostasis to allow for more functional, healthy patterns of interaction to emerge (as in family therapy) and, alternatively, additively improving functioning by building on family members' skills and competencies (implicit as skill-building interventions' theory of change). ILM can allow researchers to directly test theories of change by modeling indicators of functioning throughout the period of change to determine whether these outcomes gradually improve or show a period of disruption and variability. 
Assessment on micro timescales may be especially sensitive to detecting changes (e.g., changes in momentary experiences that have not yet accumulated to impact broader outcomes or global perceptions) and therefore especially useful for the evaluation of preventive interventions and in microtrials of intervention components (Leijten et al., 2015), which may in the short-term show small changes that cascade later in development. Examples of momentary experiential outcomes include affective quality of a parent-child interaction, child behavior, child internal states, and lability of these daily experiences.

Similarly, some hypothesized mechanisms involve day-to-day behaviors and interactions: for example, family chaos/positive family routines, calm reactions to disclosure, appropriate behavior management, emotion coaching, and responsive parenting. ILM can capture minute changes in interactions that can be expected to repeat and accumulate over longer periods of time and predict cascading changes in children and parents.

\section{The Application of Intensive Longitudinal Methods to Applied Family Research}

Theoretical perspectives on change processes that occur on a micro timescale inform what trajectories can be expected, and guide assessment timing and spacing, research questions, and data analysis. In this section I focus on measurement timing, new research questions and suggest an example study that could advance research on the intervention change process and provide valuable information for clinical practice. ILM create an opportunity to address a broad array of fascinating and nuanced research questions in applied family research.

\section{Measuring Processes on a Micro Timescale}

To best assess the dynamic processes occurring rapidly in micro timescales, participants should be assessed as the intervention is delivered to capture the beginning and course of the change process. To capture the change process, which may be complex and recursive, frequency of assessment during this period necessarily should be high-randomly sampling moments throughout the day, for example. As mentioned above, these methods closely match theories of development and theories of change of many family interventions, which suggest complex short-term change processes in parenting behaviors and family interactions such as reactivity to changes in family members' behavior, system adaptation, negative feedback loops, escalation within an interaction, disruption to homeostasis, and systematic effects of nonlinear time such as day of the week. In order to use intensive longitudinal assessment to examine macro timescale intervention efficacy on impacting dynamic characteristics and momentary experiences, participants should be assessed intensively at baseline and, at a minimum, at one post intervention burst in a multiple timescale design (Nesselroade, 1991; Sliwinski, 2008). And, attention should be paid to the timing of assessment at the micro timescale as with dynamic processes. Figure 1 shoes how frequency and timing of measurement will impact conclusions about the trajectory of a construct over micro time. That is, assessments must be matched with the rapidity of the fluctuation or change lest the within-subject variability be underestimated, and timing assessments to capture critical moments of change can help to minimize the frequency of assessments needed. To best uncover the course of change and intervention mechanisms, participants' 
dynamic characteristics and processes should be assessed as the intervention is delivered to capture change in mechanisms it occurs on a medium timescale.

Using multiple reports from each subject on hypothesized mediators and outcomes at measurement bursts before, during, and after an intervention, ILM can provide an understanding of whether parents and children behave differently on days following versus preceding intervention modules and what factors contribute to higher-functioning days. For example, for one family, on occasions when a parent's monitoring behavior is higher than usual, child behavior is improved whereas in another family, on occasions when a parent's monitoring is higher than his/her usual level of monitoring, the child acts out. In this case, the direction and magnitude of within-subject mediation of monitoring is different among families and may not represent the results of between-subject mediation. Each parent in the within-subject mediation analysis has a unique estimated parameter to explain the unique relation between their monitoring and their child's behavior (see Bolger \& Laurenceau, 2013, pp. 177-195; Bauer, Preacher, \& Gil, 2006). ILMs' density of measurement provides many data points over time for each subject, allowing for these investigations of withinsubject change; thus, ILM have the potential to paint a clearer picture of within-subject intervention mechanisms and explain how interventions work for families.

Taking these recommendations together results in the recommended protocol of assessment bursts at baseline, during, and post intervention. This protocol will cover macro timescale change using comparisons between baseline and post-intervention bursts. Additionally, this protocol will cover the medium timescale process of change within the "during intervention" burst. The researcher will need to refer to previous repeated measure studies and theory specific to the constructs of interest to determine the appropriate assessment frequency and timing in the micro timescale within each burst.

\section{An Example Intervention and Intensive Protocol}

Here, I present an example intervention and study design on which research questions in the subsequent section are based. This family treatment program that targets enforcing rules calmly, and the reduction of negative/harsh communication and escalating conflict in families that are referred. The long-term outcomes include improved child mental health and improved family functioning (e.g., parent-child closeness). The intervention targets of this program can be measured by ILM to obtain a fine-grained dynamic picture of the variability and changes in each target across time, and the relations among these aspects of functioning over time. Control families are also assessed for comparison. This could provide valuable information about the inner workings of this intervention.

An intensive repeated measures study with mothers, fathers, and children participating in this intervention might assess family members over a sampling of moments each day for two weeks at baseline, during, and post-intervention (i.e., interval or device contingent). Because the constructs of interest are highly variable throughout the day and can be context-based, assessment frequency is high at 5 assessments per day. Important to analysis strategies and for triangulating perspectives across family members, all members in a family are assessed on the same sampling schedule. Questions are rated on sliding scales of 0-100. 
At each of the moments sampled, the protocol includes items assessing intervention-targeted outcomes: family members' feelings about their family relationships (e.g., parent-child closeness) and internal states (e.g., affect, stress and distress, and internalizing symptoms) in that moment. The protocol also includes items assessing the targeted mechanisms of negative communication and escalating conflict. So that the information gathered is finegrained and specific to that conflict, details are collected at the sampled moments about the most recent conflict. Family members are also asked to rate calm rules enforcement with regard to the interval since the previous assessment. The micro timescale assessed in this study is within-day, and due to the differing variabilities across constructs, the assessment protocol differs slightly at this micro timescale for each construct (i.e., affect is momentspecific, conflict intensity is conflict-specific, and rules enforcement is interval-specific).

\section{Some Research Questions Made Possible by ILM}

Table 4 maps a selection of the research questions proposed here and examples of appropriate statistical techniques. The treatment program itself can be tested as a betweensubjects predictor of treatment effects. For example, (A) does the family treatment program stabilize within-day variability in distress, affect, and internalizing symptoms compared to control families? This question focuses on macro timescale change in micro timescale dynamic constructs. Variability as a dynamic construct in mental health may be considered instability or vulnerability, although some variability is normal responsiveness to the context. Also, (B) does the family treatment program decrease the frequency and intensity of family conflict events over time, especially between child and his/her parent? In this research question, ILM contributes measurement benefits of frequency and intensity of conflict and may allow for within-family analysis.

Models of within-subject change might also be investigated to determine the functional form of change. During intervention, change may be non-existent, smooth or fluctuating. Interactions between parents and their child may fluctuate more during periods of change in parenting as an indicator of system disequilibrium. (C) Does practice of other new skills disrupt family homeostasis resulting in a period of fluctuation in a family's level of adaptive functioning? For example, a parent who has not enforced rules before may be met with a child's backlash or confusion as parent and child pave new patterns of interaction. Alternatively or perhaps co-occurring, (D) do families in the intervention program show a smooth increase in the average rating of closeness over the weeks of the intervention?

For family members assigned to the intervention, the path of change among family members can be examined. Within the coparenting subsystem, (E) does the father's change in parenting such as beginning to enforce rules calmly influence the mother's use of that skill on the following day? Or vice versa? Intervention effects may evolve in recursive ways, with changes in some family members influencing or eliciting change in other family members. ILM provides the temporal specificity needed to determine the influence among family members on change processes.

Researchers can examine the relation among constructs over time for families assigned to the intervention. For example, intensive assessment of the intensity of family conflicts and of child internal states allows one to understand how these co-occur over time for each child. 
Beyond covariation, $(\mathrm{F})$ on days when a child reports having more intense conflict with his/her mother, the child may experience more distress. Intervention studies can investigate (G) whether treatment leads to decoupling of conflict and symptoms such that family conflict does not lead to mental health problems. Alternately, $(\mathrm{H})$ a decrease in conflict intensity may mediate an intervention's decrease in mental health problems. Practice of new skills $i$ s the beginning of generalization and change, so investigating the application of new skills at home may provide insight into the change process. For example, (I) on days when a parent practices enforcing rules calmly, is the relationship with his/her child affectively more positive and closer?

Analysis-Common approaches to analyzing intensive data include variations of the multilevel model. Multilevel within-subject mediation and within-subject causal models are common methods used to model dynamic mechanisms and mediating processes (Bauer, Preacher, \& Gil, 2006; Nezlek, 2001; Schwartz \& Stone, 2007). In a basic research analysis focused on these within-subject associations, Telzer \& Fuligni (2009) found that on days when adolescents spent more time helping family members, they were happier, an association that was accounted for by higher feelings of role fulfillment on those days and was stronger for adolescents with better family relationships. Multivariate time-series models such as bivariate (i.e., dual or paired) multilevel models are common methods used to model linkages among family members' change processes because outcomes of both members of a dyad can be modeled simultaneously in a single model (Laurenceau \& Bolger, 2012; e.g., Granic, Hollenstein, Dishion \& Patterson, 2003; Saxbe \& Repetti, 2010). The heterogeneous variance multilevel model allows predictors of variance in the outcome (as opposed to predictors of level). For example, this can be used to test the hypothesis that families attending the intervention show more day-to-day variance during the intervention as a time of disruption and change compared to non-attending families (i.e., not experiencing disruption; e.g., Hedeker, Mermelstein, \& Demirtas, 2012).

In determining the functional form of change, a large and increasing variety of mathematical equations are available, which allows the flexibility to test specific hypotheses and parameters of growth or change and with varying purposes such as summarizing the shape of change across a sample, and determining patterns in variability. Some of the most commonly used techniques include nonlinear multi-level models (e.g., Fritz, 2014; Ram \& Grimm, 2007; Teachman, 2014), dynamic models (e.g., Chow, Mattson, \& Messinger, 2014; Ram, Shiyko, Lunkenheimer, Doerksen, \& Conroy, 2014), or using statistical summaries of within-subject (i.e., intraindividual) variability (c.f., Ram \& Gerstorf, 2009).

\section{An Example Study to Build on Prior Research}

An excellent example of using ILM to inform the process of intervention change and the role of within-subject mechanisms that contribute to change would be to supplement the exciting qualitative work that has been done by Holtrop, Parra-Cardona, and Forgatch (2014) on the processes of change experienced by parents using the Parent Management Training- Oregon (PMTO) model. Holtrop and colleagues used sequential coding of parents' interviews on their experiences with PMTO based on grounded theory to examine the process of change. They found that attempting to change, appraising the PMTO parenting 
strategies, and applying the skills were stages of parents' reports of change. Appraising happened throughout the learning process and fed into future attempts to use skills. In the long term, parents often reported inconsistent use of PMTO strategies because they no longer need such serious strategies to manage their child's behavior (Holtrop et al.). This type of study provides an important foundation for more deeply understanding the process of change, including its cyclical nature, and influences on change such as how parents evaluate the methods taught in a program, how parents decide whether to use skills and whether to adapt them, and when to stop using skills.

To more fully examine the hypotheses about PMTO families' change processes, it might be useful to collect intensive data in situ before, during, and after the intervention. By collecting information while these change processes occur in daily life, ILM can both corroborate and add temporal specificity to the change process described by Holtrop and colleagues. To verify parents' sense that attempting to change and appraising skills influenced skill use, an intensive repeated measures study could empirically investigate: what time-varying or stable factors influence the use of skills on subsequent days? To verify parents' sense of not needing skills as the reason for discontinuing skill use, an intensive repeated measures study could empirically investigate: what factors precipitate a decrease in skill use-improved child behavior, parent stress, changes in context? Expanding on their line of questioning, research questions might be, Is parents' skill use consistent day-to-day, or does it show significant variability? Does appraisal of skills change over time: during the session, throughout the week, through the duration of the intervention? Does the use of a skill (successful vs. unsuccessful use) impact the appraisal of that skill? Is the use of skills more common on days that the child goes to school, the child misbehaves, the parent feels less stressed? Is the child better behaved on days following high parent skill use?

\section{Use of Intensive Longitudinal Methods in Clinical and Prevention Practice}

ILM can be used to monitor treatment success by assessing constructs of targeted change throughout implementation (Bai \& Repetti, 2015; Chorpita et al., 2010; Trull \& EbnerPriemer, 2009). Throughout a program, practitioners can use participants' frequent reports to improve the likelihood of treatment success (Trull \& Ebner-Priemer, 2013). For example, review of and feedback on participants' intensive data may boost treatment effects (c.f., Rofey et al., 2010). Support can be initiated with parents one-on-one as a second-line additive intervention to overcome barriers such as low motivation when intensive data suggest the parent may be less likely to respond to treatment (c.f., Burns et al., 2011). Additionally, practitioners can conduct adjunctive one-on-one sessions with parents who may be struggling in order to reinforce skills or provide additional treatment; for example, coaching parents on skills that are reported infrequently may help parents practice and improve these skills. Coaching has been an effective method in eliciting parents' behavior change in intervention programs (Barnett, Niec, \& Acevedo-Polakovich, 2014; Guttentag, 2014). More broadly, by monitoring outcomes such as psychological symptoms, experiences, and behavior along with their contexts, participants provide more accurate information to practitioners about the patterns and covariations among these occurrences allowing practitioners to develop better treatment plans (Myin-Germeys, Birchwood, \& 
Kwapii, 2011). Intensive data could then be used to track outcomes and evaluate the effectiveness of additional support provided to improve treatment success.

\section{Related Current Applications of Intensive Longitudinal Methods}

In our own work, we have utilized daily assessments during a family intervention and found that attendance was positively related to the day-to-day variability in reports of parenting, potentially reflecting the disruption and change process that occurs with a family system's exposure to an intervention (Bamberger, Ram, Greenberg, \& Fosco, in preparation). Besides this single application to family intervention evaluation, researchers have begun using ILM across many fields of research. Here, I summarize some of those most closely connected to applied family research-intervention evaluation and basic family research, which can guide future applications of ILM to family intervention evaluation in the absence of more directly related studies.

Evaluation of interventions on a micro timescale-In a similar manner to the above advances in basic family research, ILM can be leveraged to understand families as they undergo change in an intervention (Bolger \& Laurenceau, 2013; Smyth \& Stone, 2003). However, ILM have not been employed with family interventions to delve deeper into family outcomes or processes of change. Intervention studies outside the area of family research have been quite promising in using ILM to advance scientific knowledge. Most studies have used ILM to assess intervention effects on momentary and dynamic experiences, but other contributions include novel approaches to modeling change processes and prediction of treatment impact using momentary experiences at baseline.

In therapy and behavioral interventions, intensive repeated measures studies have investigated intervention effects and tested theories of change. One study evaluated a drinking intervention (moderation training) with adults and revealed that ILM constituted a feasible way to evaluate this intervention and found that, among other variables, episodespecific mood predicted excessive drinking during a drinking episode (R. L. Collins, Morsheimer, Shiffman, Paty, Gnys, \& Papandonatos, 1998). In an evaluation of a structured writing intervention on objective arthritis and asthma indicators, Smyth and colleagues found that intensively assessed mood and health behaviors did not mediate the significant intervention effects at either the between-subjects or within-subject level (reported in Smyth \& Stone, 2003). In a comparative treatment study for anxiety in children and adolescents, Cognitive Behavioral Therapy (CBT) but not Child-Centered Therapy was associated with lower peak negative affect within the hour prior to random assessment calls at the end of treatment (week 12 and post-treatment) compared to week 4 of treatment (Silk et al., in press).

Recent medical studies have also shown that ILM advances evaluation science. In treatment of child and adolescent depression and anxiety, one study found that momentary positive affect and the ratio of momentary positive to negative affect on the week before treatment predicted treatment impact in symptom severity, while self-reported (retrospective/trait) symptoms at baseline did not (Forbes et al., 2012). This study demonstrates the validity and added benefit of ILM and the current experiences that they assess. 
Perhaps the type of interventions most tied to intensive assessment in the research literature is ecological momentary interventions (i.e., EMI, mHealth interventions) - interventions delivered in situ and in the moment such as messages delivered on smart phones (Heron \& Smyth, 2010; Riley et al., 2011). Intensive assessment is inherent in many EMIs because information from the participant is used to inform the delivery of intervention messages, especially in individualized and tailored EMI (Elfeddali, Bolman, \& de Vries, 2012; Rotheram-Borus et al., 2012). ILM have been applied to the evaluation of EMI with varying degrees of sophistication, and certainly can be leveraged to evaluate these programs to a greater degree (Heron \& Smyth, 2010; e.g., Voogt, Kuntsche, Kleinjan, \& Engels, 2014).

Although ILM have not been applied to the evaluation of family interventions, the application of ILM to other types of interventions shows great potential and feasibility for this avenue of research.

Research with families on a micro timescale-Family researchers have begun applying ILM to basic family research (for a recent review see Repetti, Reynolds \& Sears, 2015). Studies have reported good compliance and feasibility of ILM with a variety of samples including with parents and children; for example, a 56-day (two-month) protocol of five-minute daily diaries was feasible for parents and their 8-13 year olds (Robles, Reynolds, Repetti, \& Chung, 2013).

Family research has shown that ILM capture valid and important daily family interactions that are related to outcomes. For example, studies have shown that family tension and conflict are interrelated among family subsystems and relate to outcomes such as distress and positivity. Tense interactions between parents spill over to parents' tense interactions with children the next day (Almeida, Wethington, \& Chandler, 1999), and this parentadolescent conflict is the mediator by which day-to-day interparental conflict leads to adolescent emotional distress (Chung, Flook \& Fuligni, 2009). On days when adolescents experience interparental conflict, adolescents are more distressed than on other days (Chung et al., 2009). This research illuminates the path by which interparental subsystem functioning may impact the adolescent on a day-to-day basis. These findings reflect a broader principle of spillover (especially of negativity) from one family subsystem to another, which can only be modeled with intensive longitudinal data collected once or more daily (Larson \& Almeida, 1999; Repetti, 1987).

Research has also revealed time-trends in family conflict along with time-varying interplay of conflict and well-being among family members. Almeida and McDonald (1998) found that parent-adolescent tension shows rhythms in frequency across days of the week, and the occurrence of tension was predicted by parents' work-related and home-related stress, especially mothers' home stress. Within the family system, parent-child tension was similar for both parents, determined for both parents by mothers' stress and spouse-child tension. It seems that day-to-day conflicts are sources of distress for parents, more so than for adolescents (Steinberg, 2001), and the repetition of conflicts impacts mothers' mental health (Silverberg \& Steinberg, 1987 as cited in Steinberg, 2001). In addition, girls who report a large proportion of days getting along with their family have low levels of internalizing comparable to those of boys (Telzer \& Fuligni, 2013). Broadly, this line of research 
demonstrates that there are reciprocal effects between the adolescent and the parent within the family system; because such feedback loops are rapid processes, they can only be modeled with intensive longitudinal data collected at the interaction or daily level.

Other studies focus on interactional dynamics within the family (Fuligni, 2014). For example, between conflict events, wives' positivity was not predictable, but husband's positivity was predicted by wives' positivity from the previous conflict episode (Schermerhorn Chow, \& Cummings, 2010). Ferrer and Helm (2013) have shown that couples co-regulate their heart rates and respiration rates in laboratory tasks, and that the degree to which females' physiology changes to match their partner's physiology is associated with the degree to which their daily reported affect changed to match their partner's. This synchrony occurs as persons become more similar and change together across time and can be modeled with many data points from each person in a couple.

In summary, basic research with families using ILM is beginning to lead to greater precision and specificity in our understanding of family dynamics, interactions, and the ways in which they impact family members' well-being and development. ILM expands the research agenda by allowing measurement of these rapid family dynamics.

\section{Challenges and Considerations}

There are many considerations in designing an intensive repeated measures study to achieve the benefits of ILM and address new research questions. Decisions among assessment protocols-whether interval-, event-, and/or signal-contingency is used and frequency of assessment in the micro timescale-should be made to capture within-subject variability in the constructs of interest (e.g., are processes of interest occurring day-to-day or interactionto interaction?). This should be balanced with participant burden to ensure that adequate data are collected but not so much data as to cause undue burden to participants. Timing of assessments bursts should be conducted at theoretically relevant time points in the macro timescale as well in order to further our understanding of change processes (e.g., consideration of the expected timing of change and maintenance or fade out).

There are substantial advantages to using ILM in applied family research, but, there are challenges that come with their use. The number and frequency of assessments in intensive repeated measures studies calls for frequent introspection and recall, which could result in participant reactivity (Repetti, Reynolds, \& Sears, 2015; Bolger \& Laurenceau, 2013). Reactivity results to the extent that participants are influenced by their own increased attention to and evaluation of the assessed constructs and respond by changing their behavior-such as decreasing or increasing the number of "events" they experience in event-contingent designs, or attempting to enact more personally acceptable behavior. This is not a phenomenon of changing responses (e.g., social desirability bias) but rather changing actual behavior. There is not much evidence published in support or refute of reactivity effects in the context of intensive repeated measures studies, but reactivity may be more likely in study designs that involve several assessments per day. Some researchers suggest that ILM can be perceived as unintrusive and may not alter daily life (Ho \& Intille, 2005; Smyth \& Heron, 2014). One study had mixed conclusions about whether eventcontingent assessment multiple times per day resulted in greater reactivity than end of day 
assessment in a two-week protocol (Merrilees, Goeke-Morey, \& Cummings, 2008); specifically, no differences in husbands' or wives' behavior were noted in observation of conflict tasks completed after the reporting period, yet husbands' daily reports of global marital quality declined over time in only the event-contingent group. Runyan and colleagues (2013) have used ILM as an intervention targeting self-awareness through repeated introspection, yet increased self-awareness does not necessarily lead to changes in behavior, and even in this study decreased compliance across the study could have contributed to statistical differences in reported behavior. On one hand, repeated assessment and resulting reactivity could be viewed as a comparatively easy and potentially effective intervention to the extent that reactivity supports positive change; on another hand, this effect calls into question the external validity of the depiction of daily life that can be garnered from ILM.

In addition, although ILM can be perceived as unintrusive, the timing and frequency of data collection may cause significant participant burden, which may result in issues with compliance, fatigue, or drop-out (Repetti, Reynolds, \& Sears, 2015; Bolger \& Laurenceau, 2013). In device-contingent and interval-contingent designs, time-stamping completed responses allows researchers to evaluate participant compliance with the assessment protocol; on-time completion rates are usually acceptable in these designs especially with additional incentives and other strategies to increase compliance, but compliance is difficult to determine in event-contingent designs because event occurrence often cannot be verified when surveys are not completed. Also problematic, compliance may wane over the study period due to fatigue. Because of this risk, measures must be brief and questions must not be cognitively demanding (Stone \& Shiffman, 1994). As a result, lengthy standardized questionnaires developed for retrospective data collection cannot be used in intensive repeated measures studies. Yet, current states and experience can be captured accurately and quickly. With few, brief items per construct and sliding scales with large ranges, withinsubject variability is captured most accurately, making this type of measurement appropriate for the research questions that ILM addresses. In the context of an intervention study, we have found that intensive daily data collection is acceptable for a volunteer group of parents based on post-study satisfaction surveys and produces useful data demonstrating the complexity and nature of the change process (Bamberger, Ram, Greenberg, \& Fosco, in preparation).

Because participants will be responding to the same questions time and again, training participants to use new technologies and interfaces along with the meaning of assessment questions and, in event-contingent designs, definitions of events is useful for data quality (Stone \& Shiffman, 1994). Yet, training each participant may be logistically difficult, and some aspects of a protocol in which adults would need training may be even more difficult for young children.

It is yet to be seen whether children younger than about eight years of age can complete these assessment protocols, which could be of concern for family researchers who focus on families with young children. Otherwise, children are often familiar with the devices and technology that is often used for ILM, and guided interaction can be used to familiarize children with new interfaces (Plowman \& Stephen, 2007). Additionally, because of the low 
cognitive demands of the brief measures used in ILM, there is reason to believe that even young children may be able to complete thoughtfully constructed protocols.

ILM capture information about all or a sample of moments in the participant's life, creating an abundance of data. Yet, in predicting long-term developmental outcomes, it cannot be assumed that each moment is of equal importance in its contribution. Thus, researchers will be tasked with using lots of available data to obtain meaningful summaries of experience that relate to these long-term outcomes. This task may prove challenging, and for the purpose of predicting long-term outcomes, may not be more useful than asking a participant to summarize their experience into retrospective reports of constructs of interest. While it remains to be seen whether intensive longitudinal data is useful and efficient for this type of research, intensive longitudinal assessment is very likely to be ground-breaking in its usefulness in modeling dynamic characteristics and processes.

Depending on the technology and interface used, compliance measures necessary, and training involved in a given ILM protocol, this method of data collection can be costly. Thus, ILM should be used to answer research questions that require this method.

\section{Resources for Designing Intensive Repeated Measures Studies and Using Intensive Longitudinal Data}

Researchers interested in applying ILM to evaluating intervention programs can consult several noteworthy sources for practical considerations of study design, measurement, and data analysis.

Stone, Shiffman, Atienza, and Nebeling (2007) introduce the historical development of ILM (here, "EMA") with a useful summary of rationale, benefits, and challenges of ILM. There is a brief guide on analysis of intensive longitudinal data. The editors also include examples of research using ILM in behavioral and medical health fields. Perhaps most useful to researchers new to using ILM is a chapter discussing study design, which details the considerations of matching the time sampling protocol to the timing of experiences of interest.

Mehl and Conner (2012) have edited a volume that is entitled and can indeed be thought of as a handbook of ILM. They include theoretical rationales for collecting data in the real world, in real time, and within person (many repeated measures), which I have covered here as these benefits of ILM apply more specifically to family and developmental theories and the goals of applied family research. The chapters also go into great depth on the wide variety of protocols and technology that can be used in ILM and considerations for choosing among these in designing a study, which I have only covered cursorily. Also covered are a wide variety of statistical modeling strategies that can be applied to intensive longitudinal data structures, which go beyond the examples that I have included throughout this paper. Some applied family researchers may also find useful the chapters on the application of ILM to specific fields of psychology, especially perhaps, chapters on the application to the fields of close relationships, developmental psychology, and clinical psychology. 
Diehl, Hooker, \& Sliwinski (2015) provide a conceptual focus on the dynamic constructs across the lifespan that can be assessed with ILM, reviewing previous work that examines within-subject variability in these constructs. Later chapters also cover the developmental significance of within-subject variability, including its relation to long-term developmental change and its role in understanding complex developmental processes.

Bolger and Laurenceau (2013) and Walls and Schafer (2006) have focused on the analysis of intensive longitudinal data. Bolger and Laurenceau briefly describe the method and types of study designs. The authors focus on analysis in several chapters that serve as a step-bystep guide to implementing common analyses with intensive longitudinal data. They include code for SPSS, SAS, R, and other statistics programs, write-ups of results, and recommended further reading for each analysis model. Walls and Schafer provide a chapter each on advanced modeling strategies for intensive longitudinal data, including state-space modeling and systems modeling among many others. Ram and Gerstorf (2009) also discuss analysis, focusing on within-subject variability and many statistical methods to explore it.

These and additional noteworthy and applicable resources can be found in the References section indicated by asterisks.

\section{Recommendations}

Evaluating change processes in interventions requires methodological and analytical innovation because the established long intervals between baseline and post assessments make detection of mechanisms and processes difficult (Kazdin, 2007). Additionally, innovation is necessary to measure dynamic characteristics and processes and link these micro timescale constructs with intervention-induced change over macro timescales. ILM, especially multiple timescale design, is an innovation that can be applied with intervention RCT participants to make these advancements.

In carrying out multiple timescale designs, I recommend that applied family researchers (1) assess participants in their evaluation studies using ILM in bursts at baseline, throughout the intervention, and after the intervention at theoretically important time points in the macro timescale to capture the intervention change process. Specifically, separating bursts by two weeks to four weeks may allow researchers to capture these periods while giving participants a break between bursts to minimize burden, though the ideal timing, length, and separation of bursts may differ among intervention curricula.

I recommend that applied family researchers (2) consider the theoretical and empirical micro timescales of change and variability for each construct when determining the intensive assessment schedule to ensure that enough data is collected to model within-subject processes and dynamic constructs while minimizing participant burden. Because the ideal frequency of assessments within each burst is highly dependent on both the constructs of interest and the frequency of delivery and the theory of change of the intervention curriculum—daily or more frequent—researchers must be very thoughtful about this aspect of the study design. 
I recommend that applied family researchers (3) employ training, compliance monitoring, and incentives to maximize participant compliance in intensive repeated measure study designs. Training should also be used to ensure comprehension of the questions and rating scales. There are few things worse than investing resources for seven weeks of daily data and receiving only a few days' surveys from some participants. The reliability of withinsubject estimates of variability and change can be low for participants giving so few points of information over time. Making the device and interface easily accessible goes a long way toward compliance. Active efforts to train, touch base with participants, and communicate that the data matter to the researchers are best practice because they increase compliance even further.

The most useful applications of ILM utilize the many data points that each participant provides, answering questions that cannot be addressed by other (less intensive) study designs. I recommend that applied family researchers (4) use ILM to investigate withinsubject processes of change during and after an intervention (e.g., mediators, influences among family members, shape of change). Research questions about within-subject change utilize the time-structure of participants' data points and may elucidate the process by which interventions have their effects on families.

I recommend that applied family researchers (5) use ILM to investigate intervention effects on within-subject variability (e.g., lability, sensitivity, homeostasis). Research questions about within-subject variability utilize summaries of the variability in participants' data. Besides or instead of impacting the overall level of positive affect, for example, interventions may decrease lability in affect from unhealthy to healthy levels. Changes in variability may indicate increases or decreases in the stability of a family system. By addressing research questions on within-subject change and variability, studies using ILM and corresponding statistical methods to evaluate family interventions will contribute substantially to the understanding of intervention effects and change processes in families.

\section{Acknowledgments}

The author would like to especially thank Nilam Ram for his mentorship in intensive longitudinal methods and their analysis and Mark Greenberg for helpful comments on this manuscript. The author also thanks J. Douglas Coatsworth for his accommodation of the author's intensive longitudinal data collection overlaid on his evaluation of a family preventive intervention. The author gratefully acknowledges the support provided by the National Institutes of Health's National Institute on Drug Abuse (T32 DA0176, F31 DA038409) and the Bennett Pierce Prevention Research Center. The content is solely the responsibility of the author and does not necessarily represent the official views of the funding agency. Thanks to Nilam Ram, David Lydon, and Lizbeth Benson for their helpful comments on a previous version of this manuscript.

\section{References}

*. Almeida DM, McDonald D. Weekly rhythms of parents' work stress, home stress, and parentadolescent tension. New Directions for Child and Adolescent Development. 1998; 82:53-67. [PubMed: 12794951]

Almeida DM, Wethington E, Chandler AL. Daily transmission of tensions between marital dyads and parent-child dyads. Journal of Marriage and the Family. 1999; 61:49-61.

*. Bai S, Repetti RL. Short-term resilience processes in the family. Family Relations. 2015; 64:108119.10.1111/fare.12101 [PubMed: 26246651] 
Bamberger, KT.; Coatsworth, JD. An expanded conceptual model of participant engagement for prevention and intervention research. Poster presented at the Society for Prevention Research 21st Annual Conference; San Francisco, CA. May. 2013

Bamberger KT, Coatsworth JD, Fosco GM, Ram N. Change in participant engagement during a family-based preventive intervention: Ups and downs with time and tension. Journal of Family Psychology. 2014; 28:811-820. [PubMed: 25383795]

Bamberger K, Ram N, Greenberg MT, Fosco GM. A test of family system change in a prevention program: Heterogeneous variance in intensively assessed day-to-day functioning. in preparation.

Barnett ML, Niec LN, Acevedo-Polakovich ID. Assessing the key to effective coaching in ParentChild Interaction Therapy: The therapist-parent interaction coding system. Journal of Psychopathology Behavioral Assessment. 2014; 36:211-223.10.1007/s10862-013-9396-8 [PubMed: 24839350]

Barrera M Jr, Sandler IN. Prevention: A report of progress and momentum into the future. Clinical Psychology: Science and Practice. 2006; 13(3):221-226.

Bauer DJ, Preacher KJ, Gil KM. Conceptualizing and testing random indirect effects and moderated mediation in multi-level models: New procedures and recommendations. Psychological Methods. 2006; 11:142-163. [PubMed: 16784335]

Birditt KS, Nevitt MR, Almeida DM. Daily interpersonal coping strategies: Implications for selfreported well-being and cortisol. Journal of Social and Personal Relationships. 2014; 32:687706.10.1177/0265407514542726 [PubMed: 26213435]

Bjørknes R, Kjøbli J, Manger T, Jakobsen R. Parent training among ethnic minorities: Parenting practices as mediators of chance in child conduct problems. Family Relations. 2012; 61:101-114.

*. Bolger, N.; Laurenceau, J-P. Intensive Longitudinal Methods: An introduction to diary and experience sampling research. New York, NY: Guilford Press; 2013.

*. Bolger N, Davis A, Rafaeli E. Diary methods: Capturing life as it is lived. Annual Review of Psychology. 2003; 54:579-616.10.1146/annurev.psych.54.101601.145030

Bronfenbrenner U. Ecology of the family as a context for human development: Research perspectives. Developmental Psychology. 1986; 22:723-742.

Bronfenbrenner, U.; Morris, PA. The ecology of developmental processes. In: Damon, W., editor. Handbook of Child Psychology. 5. New York: Wiley; 1997. p. 993-1028.

Burns MN, Begale M, Duffecy J, Gergle D, Karr CJ, Giangrande E, Mohr DC. Harnessing context sensoring to develop a mobile intervention for depression. Journal of Medical Internet Research. 2011; 13(3):e55.10.2196/jmir.1838 [PubMed: 21840837]

Caruthers AS, Van Ryzin MJ, Dishion TJ. Preventing high-risk sexual behavior in early adulthood with family interventions in adolescence: Outcomes and developmental processes. Prevention Science. 2014; 15:S59-S69.10.1007/s11121-013-0383-9 [PubMed: 23536124]

Chorpita BF, Reise S, Weisz JR, Grubbs K, Becker KD, Krull JL. The Research Network on Youth Mental Health. Evaluation of the Brief Problem Checklist: Child and caregiver interviews to measure clinical progress. Journal of Consulting and Clinical Psychology. 2010; 78:526536.10.1037/a0019602 [PubMed: 20658809]

Chow, S-M.; Mattson, WI.; Messinger, DS. Representing trends and moment-to-moment variability in dyadic and family processes using state-space modeling techniques. In: McHale, SM.; Amato, P.; Booth, A., editors. Emerging Methods in Family Research; National Symposium on Family Research; New York, NY: Springer; 2014. p. 39-55.

Chung GH, Flook L, Fuligni AJ. Daily family conflict and emotional distress among adolescents from Latin American, Asian, and European backgrounds. Developmental Psychology. 2009; 45(5): 1406-1415. [PubMed: 19702401]

Collins LM. Analysis of longitudinal data: The integration of theoretical model, temporal design, and statistical model. Annual Review of Psychology. 2006; 57:505-528.

Collins RL, Morsheimer ET, Shiffman S, Paty JA, Gnys M, Papandonatos GD. Ecological momentary assessment in a behavioral drinking moderation training program. Experimental and Clinical Psychopharmacology. 1998; 6(3):306-315. [PubMed: 9725114] 
Conner TS, Barrett LF. Trends in ambulatory self-report: The role of momentary experience in psychosomatic medicine. Psychosomatic Medicine. 2012; 74:327-337.10.1097/PSY. 0b013e3182546f18 [PubMed: 22582330]

Csikszentmihalyi M, Larson R, Prescott S. The ecology of adolescent activity and experience. Journal of Youth and Adolescence. 1977; 6:281-294. [PubMed: 24408457]

Curtis NM, Ronan KR, Borduin CM. Mutlisystemic Treatment: A meta-analysis of outcome studies. Journal of Family Psychology. 2004; 18:411-419.10.1037/0893-3200.18.3.411 [PubMed: 15382965]

DeGarmo DS, Patterson GR, Forgatch MS. How do outcomes in a specified parent training intervention maintain or wane over time? Prevention Science. 2004; 5(2):73-89. [PubMed: 15134313]

*. Diehl, M.; Hooker, K.; Sliwinski, MJ., editors. Handbook of Intraindividual variability across the life span. New York, NY: Routledge; 2015.

Dishion, TJ.; Stormshak, EA. Intervening in children's lives: An ecological, family-centered approach to mental health care. Washington, DC: American Psychological Association; 2007.

Durlak JA, Wells AM. Primary prevention mental health programs for children and adolescents: A meta-analytic review. American Journal of Community Psychology. 1997; 25(2):115-152. [PubMed: 9226860]

Elfeddali I, Bolman C, de Vries H. SQ4U-A computer tailored smoking relapse prevention program incorporating planning strategy assignments and multiple feedback time points after the quitattempt: Development and design protocol. Contemporary Clinical Trials. 2012; 33:151-158. [PubMed: 22015574]

Ferrer E, Helm JL. Dynamical systems modeling of physiological coregulation in dyadic interactions. International Journal of Psychophysiology. 2013; 88:296-308.10.1016/j.ijpsycho.2012.10.013 [PubMed: 23107993]

Forbes EE, Stepp SD, Dahl RE, Ryan ND, Whalen D, Axelson DA, Silk JS. Real-world affect and social context as predictors of treatment response in child and adolescent depression and anxiety: An ecological momentary assessment study. Journal of Child and Adolescent Psychopharmacology. 2012; 22:37-47.10.1089/cap.2011.0085 [PubMed: 22339611]

Forgatch, MS. Parenting through change: A programmed intervention curriculum for groups of single mothers. Eugene: Oregon Social Learning Center; 1994.

Forgatch MS, Patterson GR, Degarmo DS, Beldavs ZG. Testing the Oregon delinquency model with 9 year followup of the Oregon Divorce Study. Development and Psychopathology. 2009; 21:637660.10.1017/S0954579409000340 [PubMed: 19338702]

Fosco, GM.; Bumbarger, B.; Bamberger, KT. Thinking systematically for enduring family change. In: Van Ryzin, MJ.; Kumpfer, KL.; Fosco, GM.; Greenberg, MT., editors. Family-based prevention programs for children and adolescents: Theory, research, and large-scale dissemination. New York: Taylor \& Francis/Psychology Press; 2015. p. 308-328.

Fosco GM, Van Ryzin M, Stormshak EA, Dishion TJ. Putting theory to the test: Examining family context, caregiver motivation, and conflict in the Family Check-Up model. Development and Psychopathology. 2014; 26:305-318.10.1017/S0954579413001004 [PubMed: 24438894]

Fritz MS. An exponential decay model for mediation. Prevention Science. 2014; 15:611-622.10.1007/ s11121-013-0390-x [PubMed: 23625557]

Fuligni, AJ. The benefits and challenges of modeling intra-family variability in dyadic and family processes using state-space modeling techniques. In: McHale, SM.; Amato, P.; Booth, A., editors. Emerging Methods in Family Research; National Symposium on Family Research; New York, NY: Springer; 2014. p. 57-69.

Granic, I.; Dishion, T.; Hollenstein, T. The family ecology of adolescence: A dynamic systems perspective on normative development. In: Adams, GR.; Berzonsky, MD., editors. Blackwell Handbook of Adolescence. Malden: Blackwell Publishing; 2003. p. 60-91.

Granic I, Hollenstein T, Dishion TJ, Patterson GR. Longitudinal analysis of flexibility and reorganization in early adolescence: A dynamic systems study of family interactions. Developmental Psychology. 2003; 39:606-617. [PubMed: 12760527] 
Grzywacz JG, Almeida DM, McDonald DA. Work-family spillover and daily reports of work and family stress in the adult labor force. Family Relations. 2002; 51:28-36.

Guttentag CL. Using Live Coaching and Video Feedback to Teach Responsive Parenting Skills: Experience from the PALS Project. Journal of Applied Research on Children: Informing policy for children at risk. 2014; 5(1):article 5. http://digitalcommons.library.tmc.edu/childrenatrisk/vol5/ iss $1 / 5$.

Hedeker D, Mermelstein RJ, Demirtas H. Modeling between-subject and within-subject variances in ecological momentary assessment data using mixed-effects location scale models. Statistics in Medicine. 2012; 31:3328-3336.10.1002/sim.5338 [PubMed: 22419604]

*. Hektner, JM.; Schmidt, JA.; Csikszentmihalyi, M. Experience Sampling Method. Thousand Oaks, CA: SAGE Publications, Inc; 2007.

Heron KE, Smyth JM. Ecological momentary interventions: Incorporating mobile technology into psychosocial and health behavior treatments. British Journal of Health Psychology. 2010; 15:1-39. [PubMed: 19646331]

Ho, J.; Intille, SS. Using context-aware computing to reduce the perceived burden of interruptions from mobile devices. CHI conference proceedings; April 2-7 2005; 2005. p. 909-918.

Holtrop K, Parra-Cardona JR, Forgatch MS. Examining the process of change in an evidence-based parent training intervention: A qualitative study grounded in the experiences of participants. Prevention Science. 2014; 15:745-756.10.1007/s11121-013-0401-y [PubMed: 23677458]

O'Connell, ME.; Boat, T.; Warner, KE., editors. Institute of Medicine. Y. Board on Children, and Families, Division of Behavioral and Social Sciences and Education. Preventing Mental, Emotional, and Behavioral Disorders Among Young People: Progress and Possibilities. Washington, D.C: National Academies Press; 2009.

Kazdin AE. Evidence-based treatments: Challenges and priorities for practice and research. Child and Adolescent Psychiatric Clinics of North America. 2004; 13:923-940. [PubMed: 15380789]

Kazdin AE. Mediators and mechanisms of change in psychotherapy research. Annual Review of Clinical Psychology. 2007; 3:1-27.

Lamborn SD, Mounts NS, Steinberg L, Dornbusch SM. Patterns of competence and adjustment among adolescents from authoritative, authoritarian, indulgent, and neglectful families. Child Development. 1991; 62:1049-1065. [PubMed: 1756655]

Larson R, Almeida DM. Emotional transmission in the daily lives of families: A new paradigm for studying family process. Journal of Marriage and Family. 1999; 61(1):5-20.

*. Laurenceau JP, Bolger N. Using diary methods to study marital and family processes. Journal of Family Psychology. 2005; 19:86-97.10.1037/0893-3200.19.1.86 [PubMed: 15796655]

Laurenceau, JP.; Bolger, N. Analyzing diary and intensive longitudinal data from dyads. In: Mehl, MR.; Conner, TS., editors. Handbook of research methods for studying daily life. New York, NY: Guilford; 2012. p. 407-422.

Leijten P, Dishion TJ, Thomaes S, Raaijmakers MAJ, Orobio de Castro B, Matthys W. Bringing parenting interventions back to the future: How randomized microtrials may benefit parenting intervention efficacy. Clinical Psychology: Science and Practice. 2015; 22:47-57.10.1111/cpsp. 12087

*. Mehl, MR.; Conner, TS., editors. Handbook of research methods for studying daily life. New York, NY: Guilford; 2012.

Merrilees CE, Goeke-Morey M, Cummings EM. Do event-contingent diaries about marital conflict change marital interactions? Behaviour Research and Therapy. 2008; 46:253-262. doi:101016/ j.brat.2007.11.007. [PubMed: 18177629]

Minuchin, S.; Fishman, HC. Family Therapy Techniques. Cambridge, MA: Harvard; 1981.

Molenaar PCM. A manifesto on psychology as idiographic science: Bringing the person back into scientific psychology, this time forever. Measurement. 2004; 2(4):201-218.

Mrazek PJ, Haggerty RJ. Reducing risk for mental disorders: Frontiers for preventive intervention research. Psychology: Science and Practice. 1994; 13(3):221-226.

Myin-Germeys I, Birchwood M, Kwapii T. From environment to therapy in psychosis: A real-world momentary assessment approach. Schizophrenia Bulletin. 2011; 37:244-247.10.1093/schbul/ sbq164 [PubMed: 21224492] 
Nesselroade, JR. The warp and the woof of the developmental fabric. In: Downs, R.; Liben, L.; Palermo, DS., editors. Visions of aesthetics, the environment \& development: The legacy of Joachim F. Wohlwill. Hillsdale, NJ: Lawrence Erlbaum Associates; 1991. p. 213-240.

Nezlek JB. Multilevel random coefficient analyses of event and interval-contingent data in social and personality psychology research. Personality and Social Psychology Bulletin. 2001; 27:771-785.

Olds DL. Improving the life changes of vulnerable children and families with prenatal and infancy support of parents: The Nurse-Family Partnership. Psychosocial Intervention. 2012; 12:129_ 143.10.5093/in2012a14

Plowman L, Stephen C. Guided interaction in pre-school settings. Journal of Computer Assisted Learning. 2007; 23(1):14-21.

Poulin F, Nadeau K, Scaramella LV. The role of parents in young adolescents' competence with peers: An observational study of advice giving and intrusiveness. Merrill-Palmer Quarterly. 2012; 58(4): 437-462.

Ram, N.; Diehl, M. Multiple-time-scale design and analysis: Pushing toward real-time modeling of complex developmental processes. In: Diehl, M.; Hooker, K.; Sliwinski, MJ., editors. Handbook of Intraindividual variability across the life span. New York, NY: Routledge; 2015. p. 308-323.

*. Ram N, Gerstorf D. Time-structured and net intraindividual variability: Tools for examining the development of dynamic characteristics and processes. Psychology and Aging. 2009; 24:778791. [PubMed: 20025395]

*. Ram N, Grimm K. Using simple and complex growth models to articulate developmental change: Matching theory to method. International Journal of Behavioral Development. 2007; 31:303316.10.1177/0165025407077751

Ram, N.; Shiyko, M.; Lunkenheimer, ES.; Doerksen, S.; Conroy, D. Families as coordinated symbiotic systems: making use of nonlinear dynamic models. In: McHale, S.; Amato, P.; Booth, A., editors. Emerging methods in family research. NY: Springer; 2014. p. 19-37.

Repetti RL. Linkages between work and family roles. Applied Social Psychology Annual. 1987; 7:98127.

Repetti RL, Reynolds BM, Sears MS. Families under the microscope: Repeated sampling of perceptions, experiences, biology, and behavior. Journal of Marriage and Family. 2015; 77:126146.10.1111/jomf.12143

Riley WT, Rivera DE, Atienza AA, Nilsen W, Allison SM, Mermelstein R. Health behavior models in the age of mobile interventions: Are our theories up to the task? Translational Behavioral Medicine. 2011; 1:53-71. [PubMed: 21796270]

Robbins, ML.; Kubiak, T. Ecological momentary assessment in behavioral medicine: Research and practice. In: Mostovsky, D., editor. The Handbook of Behavioral Medicine. New York, NY: John Wiley \& Sons, Inc; 2014. p. 429-446.

Robles TF, Reynolds BM, Repetti RL, Chung PJ. Using daily diaries to study family settings, emotions, and health in everyday life. Journal of Social and Personal Relationships. 2013; 30:179188.10.1177/0265407512457102

Rofey DL, Hull EE, Phillips J, Vogt K, Silk JS, Dahl RE. Utilizing ecological momentary assessment in pediatric obesity to quantify behavior, emotion, and sleep. Obesity. 2010; 18:1270 1272.10.1038/oby.2009.483 [PubMed: 20019675]

Rotheram-Borus MJ, Swendeman D, Chorpita BF. Disruptive innovations for designing and diffusing evidence-based interventions. American Psychologist. 2012; 67(6):463-476. [PubMed: 22545596]

Runyan JD, Steenbergh TA, Bainbridge C, Daugherty DA, Oke L, Fry BN. A smartphone ecological momentary assessment/intervention "App" for collecting real-time data and promoting selfawareness. PLOS ONE. 2013; 8(8):1-9.10.1371/journal.pone.0071325

Sameroff, AJ.; Fiese, BH. Transactional regulation: The developmental ecology of early intervention. In: Shonkoff, JP.; Meisels, SJ., editors. Handbook of Early Childhood Intervention. 2. New York, NY: Cambridge University Press; 2000. p. 135-139.

Sameroff AJ, MacKenzie MJ. Research strategies for capturing transactional models of development: The limits of the possible. Development and Psychopathology. 2003; 15:613-640. [PubMed: 14582934] 
Sandler IN, Schoenfelder EN, Wolchik SA, MacKinnon DP. Long-term impact of prevention programs to promote effective parenting: lasting effects but uncertain processes. Annual Review of Psychology. 2011; 62:299-329.10.1146/annurev.psych.121208.131619

Saxbe D, Repetti RL. For better or worse? Coregulation of couples' cortisol levels and mood states. Journal of Personality and Social Psychology. 2010; 98:92-103. [PubMed: 20053034]

Schermerhorn AC, Cummings EM. Transactional family dynamics: A new framework for conceptualizing family influence processes. Advances in Child Development and Behavior. 2008; 36:187-250. [PubMed: 18808044]

Schermerhorn AC, Chow SM, Cummings EM. Developmental family processes and interparental conflict: Patterns of microlevel influences. Developmental Psychology. 2010; 46(4):869-885. [PubMed: 20604608]

Schwartz, JE.; Stone, AA. The analysis of real-time momentary data: A practical guide. In: Stone, AA.; Shiffman, S.; Atienza, AA.; Nebeling, L., editors. The science of real time data capture: Selfreports in health research. New York, NY: Oxford University Press; 2007. p. 76-116.

Schwarz, N. Retrospective and concurrent self-reports: The rationale for real-time data capture. In: Stone, AA.; Shiffman, S.; Atienza, AA.; Nebeling, L., editors. The Science of Real Time Data Capture: Self-reports in health research. New York, NY: Oxford University Press; 2007. p. 11-26.

Scott SB, Sliwinski MJ, Mogle JA, Almeida DM. Age, stress, and emotional complexity: Results from two studies of daily experiences. Psychology and Aging. 2014; 29:577-587.10.1037/a0037282 [PubMed: 25244477]

Shiffman, S. Designing protocols for ecological momentary assessment. In: Stone, AA.; Shiffman, S.; Atienza, AA.; Nebeling, L., editors. The Science of Real Time Data Capture: Self-reports in health research. New York, NY: Oxford University Press; 2007. p. 27-53.

*. Shiffman S, Stone AA, Hufford MR. Ecological momentary assessment. Annual Review of Clinical Psychology. 2008; 4:1-32.

Silk JS, Tan PZ, Ladouceur CD, Meller S, Siegle GJ, McMakin DL, Ryan ND. A randomized clinical trial comparing individual Cognitive Behavioral Therapy and Child-Centered Therapy for child anxiety disorders. Journal of Clinical Child and Adolescent Psychology. in press.

Sin NL, Graham-Engeland JE, Almeida DM. Daily positive events and inflammation: Findings from the National Study of Daily Experiences. Brain, Behavior, and Immunity. 2015; 43:130138.10.1016/j.bbi.2014.07.015

*. Sliwinski MJ. Measurement-burst designs for social health research. Social and Personality Psychology Compass. 2008; 2:245-261.10.1111/j.1751-9004.2007.00043.x

*. Smyth, JM.; Heron, KE. Ecological momentary assessment (EMA) in family research. In: McHale, S.; Amato, P.; Booth, A., editors. Emerging methods in family research. NY: Springer; 2014. p. $145-161$.

*. Smyth JM, Stone AA. Ecological momentary assessment research in behavioral medicine. Journal of Happiness Studies. 2003; 4:35-52.

Society for Ambulatory Assessment. Home. 2014. http://www.saa2009.org

Spoth R, Rohrbach LA, Greenberg M, Leaf P, Brown CH, Fagan A, Catalano RF, Hawkins JD. Addressing core challenges for the next generation of type 2 translation research and systems: The translation science to population impact (TSci Impact) framework. Prevention Science. 2013; 14:319-351.10.1007/s11121-012-0362-6 [PubMed: 23430579]

Steinberg L. We know some things: Parent-adolescent relationships in retrospect and prospect. Journal of Research on Adolescence. 2001; 11(1):1-19.

Stone AA, Shiffman S. Ecological momentary assessment (EMA) in behavioral medicine. Annals of Behavioral Medicine. 1994; 16:199-202.

*. Stone, AA.; Shiffman, S.; Atienza, AA.; Nebeling, L., editors. The Science of Real Time Data Capture: Self-reports in health research. New York, NY: Oxford University Press; 2007.

Teachman, J. Latent growth curve models with random and fixed effects. In: McHale, SM.; Amato, P.; Booth, A., editors. Emerging Methods in Family Research; National Symposium on Family Research; New York, NY: Springer; 2014. p. 3-17. 
Telzer EH, Fuligni AJ. Daily family assistance and the psychological well-being of adolescents from Latin American, Asian, and European backgrounds. Developmental Psychology. 2009; 45:1177_ 1189.10.1037/a0014728 [PubMed: 19586187]

Telzer EH, Fuligni AJ. Positive daily family interactions eliminate gender differences in internalizing symptoms among adolescents. Journal of Youth and Adolescence. 2013; 42:1498-1511. [PubMed: 23733435]

Thomas R, Zimmer-Gembeck MJ. Behavioral outcomes of Parent-Child Interaction Therapy and Triple P-Positive Parenting Program: A review and meta-analysis. Journal of Abnormal Psychology. 2007; 35:475-495.10.1007/s10802-007-9104-9

Trull TJ, Ebner-Priemer UW. Using experience sampling methods/ecological momentary assessment (ESM/EMA) in clinical assessment and clinical research: Introduction to the special section. Psychological Assessment. 2009; 21:457-462. [PubMed: 19947780]

*. Trull TJ, Ebner-Priemer U. Ambulatory Assessment. Annual Review of Clinical Psychology. 2013; 9:151-176.

Van Ryzin MJ, Nowicka P. Direct and indirect effects of a family-based intervention in early adolescence on parent-youth relationship quality, late adolescent health, and early adult obesity. Journal of Family Psychology. 2013; 27:106-116.10.1037/a0031428 [PubMed: 23421838]

Voogt CV, Kuntsche E, Kleinjan M, Engels RCME. The effect of the 'What Do You Drink' webbased brief alcohol intervention of self-efficacy to better understand changes in alcohol use over time: Randomized controlled trial using ecological momentary assessment. Drug and Alcohol Dependence. 2014; 138:89-97.10.1016/j.drugalcdep.2014.02.009 [PubMed: 24613632]

*. Walls, TA.; Schafer, JL., editors. Models for intensive longitudinal data. New York, NY: Oxford; 2006.

Weisner TS. Ecocultural understanding of children's developmental pathways. Human Development. 2002; 45:275-281.

Weisner, TS. Well being, chaos, and culture: Sustaining a meaningful daily routine. In: Evans, GW.; Wachs, TD., editors. Chaos and its influence on children's development: An ecological perspective. Decade of behavior. Washington, DC, US: American Psychological Association; 2010. p. 211-224.

Weisner, TS.; Matheson, C.; Coots, J.; Bernheimer, LP. Sustainability of daily routines as a family outcome. In: Maynard, Ashley E.; Martini, Mary I., editors. Learning in cultural context. NY: Kluwer Academic; 2005. p. 41-73.

Weisz JR, Sandler IN, Durlak JA, Anton BS. Promoting and protecting youth mental health through evidence-based prevention and treatment. American Psychologist. 2005; 60:628-648. [PubMed: 16173895] 

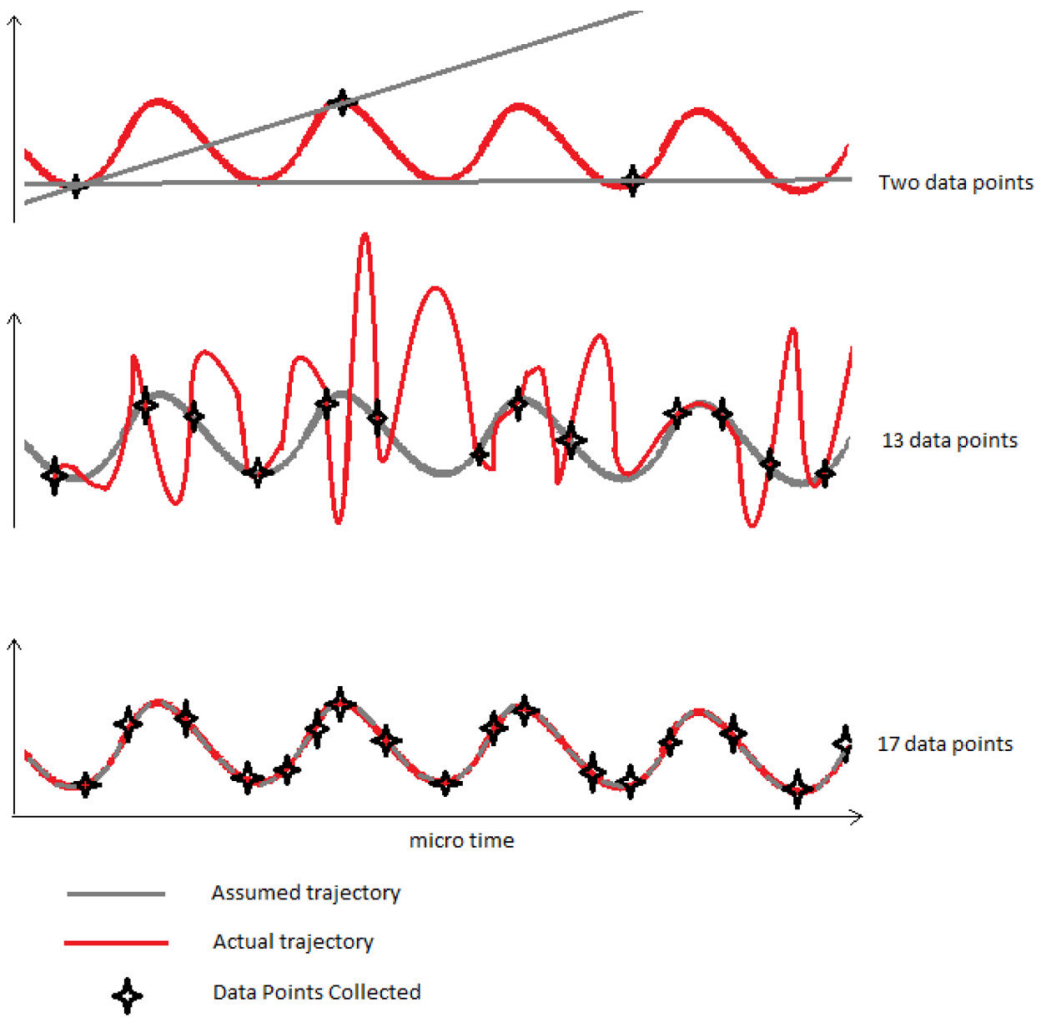

Figure 1.

Measurement timing and frequency impact the accuracy of the assumed/modeled trajectory of change or variability in the micro timescale. 


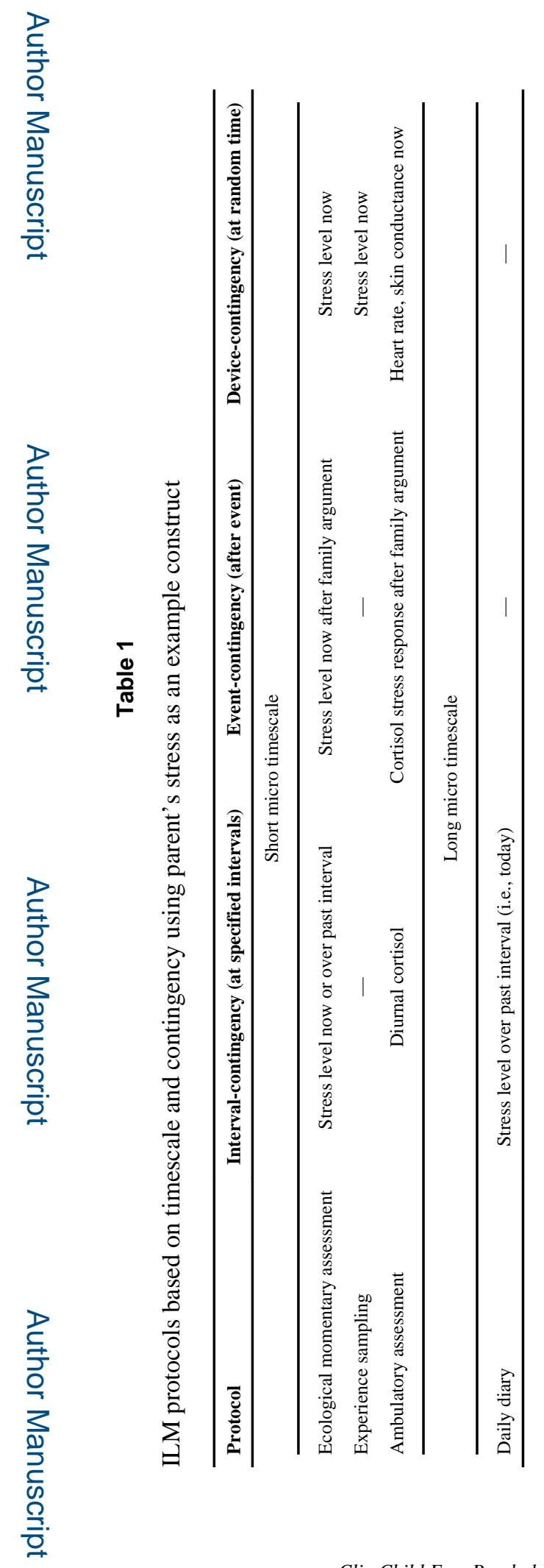

Clin Child Fam Psychol Rev. Author manuscript; available in PMC 2017 March 01. 


\section{Table 2}

Measurement and timescale benefits unique to ILM

\begin{tabular}{|c|c|c|}
\hline Retrospective/Trait Assessment & ILM & Benefits/added value of ILM \\
\hline \multicolumn{3}{|l|}{ Measurement } \\
\hline $\begin{array}{l}\text { Assesses remembering and believing selves heuristic } \\
\text { and global summaries }\end{array}$ & Experiencing self & $\begin{array}{l}\text { Captures momentary experience with } \\
\text { ecological validity and comparatively little } \\
\text { bias }\end{array}$ \\
\hline \multicolumn{3}{|l|}{ Timescale } \\
\hline $\begin{array}{l}\text { Few data points for each person, usually before and } \\
\text { after intervention }\end{array}$ & $\begin{array}{l}\text { Measurement during change Many } \\
\text { data points over micro time for each } \\
\text { person Few to many samples over } \\
\text { macro-time }\end{array}$ & $\begin{array}{l}\text { Captures specificity of temporal events } \\
\text { and the course of change in influences, } \\
\text { mechanisms, and processes }\end{array}$ \\
\hline
\end{tabular}


Table 3

Value added by ILM over retrospective assessment in understanding intervention effects

\begin{tabular}{|c|c|c|c|}
\hline New research goals & \multicolumn{2}{|r|}{ Example constructs } & $\begin{array}{l}\text { Process } \\
\text { speed }\end{array}$ \\
\hline \multicolumn{4}{|c|}{ Retrospective assessment } \\
\hline \multicolumn{2}{|c|}{ Patterns over time in characteristics } & $\begin{array}{l}\text { - Summary of experience, } \\
\text { behavior, and feelings }\end{array}$ & Slow change \\
\hline \multicolumn{4}{|c|}{ ILM } \\
\hline \multirow{2}{*}{$\begin{array}{l}\text { Patterns over time in } \\
\text { dynamic constructs } \\
\text { or momentary } \\
\text { experiences }\end{array}$} & $\begin{array}{l}\text { Dynamic } \\
\text { characteristics }\end{array}$ & $\begin{array}{l}\text { - Lability in emotions } \\
\text { - Variability in relationship quality } \\
\text { - Consistency of practice }\end{array}$ & $\begin{array}{l}\text { Slow change } \\
\text { in fast } \\
\text { process }\end{array}$ \\
\hline & $\begin{array}{l}\text { Dynamic } \\
\text { processes }\end{array}$ & $\begin{array}{l}\text { Decrease in variability } \\
\text { (approaching homeostasis) } \\
\text { - Oscillation in level of practice } \\
\text { (uptake) }\end{array}$ & Fast process \\
\hline \multicolumn{2}{|c|}{ Functional form of change } & $\begin{array}{l}\text { - } \\
\text { - } \text { Anset } \\
\text { - Duration }\end{array}$ & $\begin{array}{l}\text { Medium } \\
\text { process }\end{array}$ \\
\hline \multirow[t]{2}{*}{$\begin{array}{l}\text { Influences on within- } \\
\text { subject change }\end{array}$} & $\begin{array}{l}\text { Stable trait } \\
\text { characteristics }\end{array}$ & - Parent level of education & --- \\
\hline & $\begin{array}{l}\text { Time-varying } \\
\text { characteristics } \\
\text { or behavior }\end{array}$ & $\begin{array}{l}\text { - Engagement in session } \\
\text { - } \quad \text { Practice of skills } \\
\text { - Any dynamic construct above }\end{array}$ & --- \\
\hline
\end{tabular}

Note. Multiple timescale designs add further benefits to understanding intervention effects because they allow the identification of linkages between micro timescale dynamic characteristics and processes and macro timescale change (Ram \& Diehl, 2015).

--- indicates that process speed varies with construct 


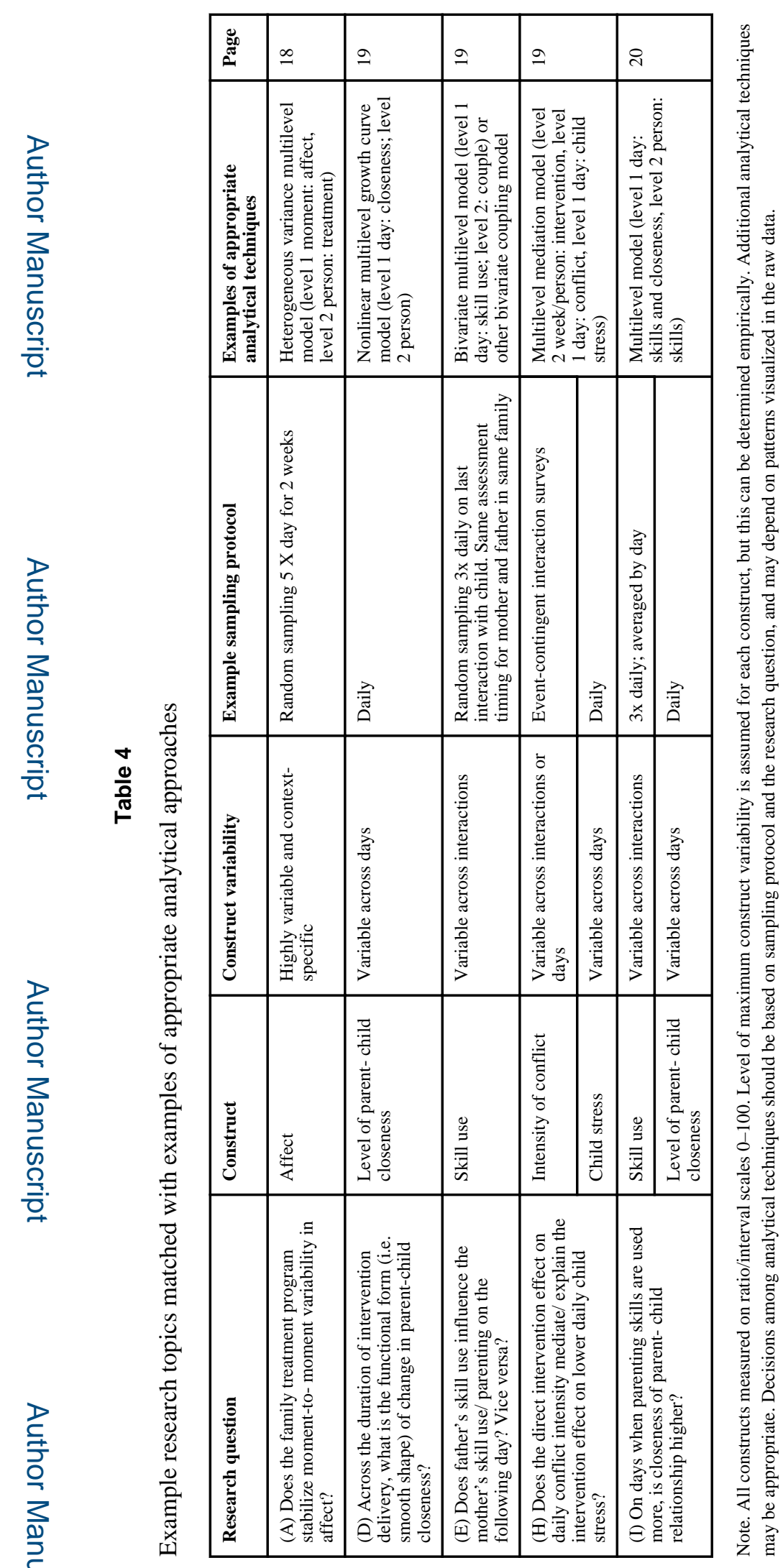

Clin Child Fam Psychol Rev. Author manuscript; available in PMC 2017 March 01. 